\title{
Aberration of French Public Health Authorities policies for the cost-assessment of microbiological diagnosis in ophthalmology
}

\author{
Pablo Goldschmidt ${ }^{\star}$, Lilia Merabet, Florence Daban, Laurence Batellier, Edgard Benveniste \\ and Christine Chaumeil
}

Laboratoire du Centre Hospitalier National d'Ophtalmologie des Quinze-Vingts, 28 rue de Charenton, 75012 ParisFrance.

Accepted 12 December, 2013

\begin{abstract}
Sensitive and specific laboratory tests adapted to ophthalmologic micro specimens are necessary for the clinical management of corneal ulcers (CU). The goal of this work is to analyze the correlation of diagnosis tests specificities with pricing policies for public laboratories. Human specimen procedures were extracted from the National Eye Center Laboratory registers in Paris, France with reimbursement values (pricing) confronting the National Social Security Services. According to cost of reactants and human resources, the official quotation for public laboratories for urinary tract diagnosis is 19 Euros (signals allowing for ruling-out negatives takes less than $2 \mathrm{~min}$ ) and less than 27 Euros for a comprehensive laboratory diagnosis of $\mathrm{CU}$, which includes testing for bacteria, fungi and protozoa (Acanthamoeba); antibacterial and antifungal susceptibility testing for positive cultures and adapted DNA extraction procedures for viral and protozoa PCR ( $>1$ hour handling). Pricing calculations produced oversimplified conclusions for $\mathrm{CU}$ diagnosis, ignoring pathology differences, diagnosis procedures, risks and sphere of action. Comprehensive revision of cost assessment policies and diagnosis pricing should be implemented for specialized laboratories to maintain the quality of public medical care and avoid interruptions in the transmission of knowledge to younger generations.
\end{abstract}

Key words: Aberration, cost assessment, laboratory diagnosis, public health, microbiological diagnosis ophthalmology.

\section{INTRODUCTION}

As a general rule it is admitted that severe eye-infections without on-time appropriate treatments may lead to visual impairment and blindness through corneal scarring, perforation or endophthalmitis. The visual function of patients presenting corneal ulcers (CU) depends upon prompt and appropriate identification of the causative agent (bacteria, fungi, protozoa, other parasites, viruses, allergens and foreign bodies among others) to administer on-time the appropriate therapeutics. Certain symptoms and signs may be associated with infectious agents (Allan and Dart, 1995). Nevertheless, a wide range of agents produce tissue damage and the lesions do not allow unequivocal recognition of the cause (Srinivasan et al., 1997; Sharma et al., 2002). Therefore, sensitive and specific laboratory tests adapted to each type of specimen are necessary for the clinical management of $\mathrm{CU}$. The goal of this work is to analyze the specificity of the laboratory procedures for the diagnosis of $\mathrm{CU}$ on 
human samples and its correlation with the pricing policies established for public laboratories.

\section{METHODS}

The procedures performed in 2013 for human specimens received at the laboratory were extracted from the National Eye Center Laboratory registers in Paris, France. The reimbursement values (pricing) for the laboratory diagnosis correspond to the amount stated by the policies of the National Social Security Services as part of the French comprehensive Social Security (SocSec).

The material sent to the laboratory should represent the replication sites of the search agents or their persistent/latent sanctuaries. Hence, laboratory results lack negative predictivevalue for specimens with inappropriate (reduced or topographically non relevant) amount of tissues.

Testing biological specimens and environmental samples need meaningful data, for which is required method optimization. Trained staff using high quality staining procedures may detect at least $40 \%$ of positive samples by direct microscopic examination if samples are representative from Protozoa replication sites.

Finally, it should be underlined that it is generally impossible to carry out systematic double checking by asking ophthalmologists for additional sampling. Intense scraping of the cornea may trigger inflammatory responses with unpredictable consequences for visual function. As a result, ophthalmologists should be warned to provide exploitable corneal specimens for laboratories performing optimized diagnosis strategies.

\section{RESULTS}

$\mathrm{CU}$ diagnosis requires materials obtained by scraping the corneal epithelia, the deep infiltrates and their periphery. Specialized laboratories should: a) assess the quality and amount of material to be tested in each specimen; $b$ ) validate the pertinence of the tests to be performed according to the clinical picture; c) decide on the appropriateness to perform certain tests according to the products that may be present on the eye surface (fluorescein, anesthetics, residual heparin, antibiotics, biocides, disinfectants and preserved eye-drops) or administered by general route before sampling (most of these agents produce false negative results if introduced into the tubes with the specimens).

The few micrograms that can be sampled should be carefully handled and divided first to smear a minimum of 2 slides, and then for the bacteria and fungi cultures. In addition, DNA from micro aliquots of the corneal scrapings should be extracted for nucleic-acid amplification tests (polymerase chain reaction for diagnosis of viruses and Protozoa infections, especially Acanthamoeba sp.).

Direct microscopic examination $(\times 1000)$ has limited negative predictive value and further testing should be performed after the first examination of stained slides. Subsequently, the immersion oil has to be eliminated and the fixed specimens must be gently de-stained (preserving cells, microbes and parasites morphology) followed by differential additional staining procedures (differential re-staining improves the visibility agents frequently included in epithelia) (Jurkunas et al., 2009 ; Sharma et al., 2002; Bharathi, 2006; Vajpayee et al., 1993). In addition, specific micro-sample procedures for germ isolation and antibiotic and antifungal susceptibility testing should be simultaneously carried-out. These procedures require qualified staff and are time consuming ( $>1 \mathrm{~h})$.

As shown by large surveys, none of the laboratory techniques can establish by itself $\mathrm{CU}$ etiologic diagnosis. In fact, there are samples for which bacterial or fungal images may or may not be found by observation and other tests may produce negative or positive for bacterial growth or no result for fungal growth. Other tests may indicate bacterial, Acanthamoeba, viral or mixed microbial growth, with unpredictable combinations (Srinivasan et al., 1997; Jurkunas et al., 2009; Sharma et al., 2002; Jones, 1981).

On the other hand, urinary tract infections (UTI) are screened using dipsticks for leukocyte esterase (LE) and nitrite. Both signals are interpreted in combination, allowing for ruling-in (both positive) and -out (both negative) urinary tract infections (Whiting et al., 2006). It was shown that dipstick negative for both LE and nitrite of a clean voided urine-bag, or nappy/pad specimen can be used to rule out UTI, excluding these samples from further investigation and establishing in less than $2 \mathrm{~min}$ negative conclusions (Wong et al., 2008; Ducharme, 2007).

A dipstick positive for either LE or nitrite provides inconclusive diagnosis and further cultures are required (negativity to the LE or the nitrite assay or both has a specificity of $92.8 \%$ ). For public laboratories Social Security establishes prices based on cost of reactants, equipment and human resources. Presently, the official quotation imposed for public laboratories (LaRocco, 1995; Brezmes et al. 2002) establishes 19 Euro for UTI diagnosis (negative results assessed in less than $5 \mathrm{~min}$ ). However, in the absence of specialists' consensus, the quotation for public laboratories for the comprehensive microbiological diagnosis of $\mathrm{CU}$ was fixed at 27 Euro. Here, negativity can be suspected only after a minimum of 1 to $2 \mathrm{~h}$ by a proficient staff following laboratory procedures including: a) slide staining, de-staining and re-staining; b) exhaustive smear microscopic examination for characterization of epithelial cells, polynuclear leucocytes, lymphocytes, macrophages and eosinophils; c) search of bacteria, fungi, protozoa and azurophile cytoplasmic epithelial-cell inclusions; d) bacterial and fungal cultures and sub cultures; e) microbial characterization of the isolates at species level; f) antibiotic and/or antifungal susceptibility testing (Journal Officiel, 2006; 2010). The diversity of parameters to be searched in the material should be highlighted here because the ratio obtained by dividing the number of tests by the mass of specimen obtained from CU is the highest in medical laboratory practice (Bharathi 2006; 
Boggild et al., 2009; Vajpayee et al., 1993).

\section{DISCUSSION}

Specialized institutions providing services to people with a broad spectrum of disorders require a direct link with specialized laboratories that are an essential part of an efficient health care system. As people with comorbidity or who have severe forms of disease require greater resources than a less complex population, additional risk adjustment should be set for institutions that do not attract the very "average" patient pathology.

The general demand for the control of health budget in France has lead financial experts to fix pricing policies because the resources of the public sector are scarce: the final goal is to lower the costs while maintaining the quality of care (Prajna et al., 2007; LaRocco, 1995; Brezmes et al., 2002).

Laboratories are often among the first sections to be targeted for budget reductions as their costs seem easily discernible. However, it has been demonstrated that dramatic reductions in laboratory testing produces disproportionately small true health cost reductions, and it is by no means certain that simply reducing test pricing will lead to a decrease in the overall health care costs (Journal Officiel, 2006). Moreover, the pricing for delivered laboratory services are not risk adjusted for "high" complexity patients.

It appears that for CU laboratory diagnosis, pricing calculations has produced oversimplified conclusions ignoring pathology differences, diagnosis procedures, risks and sphere of action. The corollary of the myopic approaches may state the entire highly specialized laboratory diagnosis as "financially non-viable" (Journal Officiel, 2006; Stentz et al., 1996; Jandreski, 1996; Das et al., 2010). Therefore reductionist assessments require comprehensive revision and emendation, first, to maintain the quality of public medical care, and second, to avoid interruption of transmission of highly specialized knowledge to younger generations.

\section{REFERENCES}

Allan BD, Dart JK (1995). Strategies for the management of microbial keratitis. Br. J. Ophthalmol. 79:777-786.

Bharathi MJ, Ramakrishnan R, Meenakshi R, Mittal S, Shivakumar C, Srinivasan M (2006). Microbiological diagnosis of infective keratitis: comparative evaluation of direct microscopy and culture results. Br. J. Ophthalmol. 90(10):1271-1276
Boggild AK, Martin DS, Lee TY, Yu B, Low DE (2009). Laboratory diagnosis of amoebic keratitis: comparison of four diagnostic methods for different types of clinical specimens. J. Clin. Microbiol. 47(5):1314-1318.

Brezmes M, Ochoa C, Eiros J (2002). Cost Analysis in a Clinical Microbiology Laboratory. Eur. J. Clin. Microbiol. Infect. Dis. 21(8): 582-588.

Das S, Sharma S, Kar S, Sahu SK, Samal B, Mallick A (2010). Is inclusion of Sabouraud dextrose agar essential for the laboratory diagnosis of fungal keratitis. Indian J. Ophthalmol. 58(4):281-286.

Ducharme J, Neilson S, Ginn JL (2007). Can urine cultures and reagent test strips be used to diagnose urinary tract infection in elderly emergency department patients without focal urinary symptoms. CJEM. 9(2):87-92.

Jandreski M (1996). What's what in laboratory finance. Clin Lab News. 22:10-11

Jones DB (1981). Decision making in the management of microbial keratitis. Ophthalmol. 88:814-820.

Journal Officiel, France JORF n`0012 du 15 janvier 2010 page 819 text $n^{\circ} 43$ Article 69 released on July 21 st 2009 for the "réforme de l'hôpital et relative aux patients, à la santé et aux territoires (loi HPST) and ordonnance (n²010-49) 15 January 2010.

Journal Officiel, France JORF $n^{\circ} 129$ du 4 june 2006 page 8515 text $n^{\circ}$ 17. Décision du 4 mai 2006 relative à la liste des actes et prestations pris en charge par l'assurance maladie Ordonnance $n^{\circ} 2010-49 \mathrm{du}$ 13 janvier 2010 relative à la biologie médicale.

Jurkunas U, Behlau I, Colby K (2009). Fungal keratitis: changing pathogens and risk factors. Cornea. 28(6):638-643.

La Rocco MT (1995). Quality and productivity in the clinical microbiology laboratory: continuous quality improvement. Clin. Microbiol. News. 17:129-131.

Prajna VN, Nirmalan PK, Saravanan S, Srinivasan M (2007). Economic analysis of corneal ulcers in South India. Cornea. 26(2):119-122.

Sharma S, Kunimoto DY, Gopinathan U, Athmanathan S, Garg P, Rao GN (2002). Evaluation of corneal scraping smear examination methods in the diagnosis of bacterial and fungal keratitis: a survey of eight years of laboratory experience. Cornea. 21(7):643-647.

Srinivasan M, Gonzales CA, George C, Cevallos V, Mascarenhas JM, Asokan B, Wilkins J, Smolin G, Whitcher JP(1997). Epidemiology and aetiological diagnosis of corneal ulceration in Madurai, South India. Br. J. Ophthalmol. 81:965-971.

Stentz F, Stevens MV, Rhodes A, Starnes S (1996). Redefining the laboratory in an era of cost constraints. Am. Clin. Lab. 14:6-8.

Vajpayee RB, Angra SK, Sandramouli S, Honavar SG, Chhabra VK (1993). Laboratory diagnosis of keratomycosis: comparative evaluation of direct microscopy and culture results. Ann Ophthalmol. 2568-2571.

Whiting P, Westwood M, Bojke L, Palmer S, Richardson G, Cooper J, Watt I, Glanville J, Sculpher M, Kleijnen J (2006). Clinical effectiveness and cost-effectiveness of tests for the diagnosis and investigation of urinary tract infection in children: a systematic review and economic model. Health Technol. Assess. 10(36):1-154.

Wong HF, Lee LC, Han HC (2008). Cost-effective screening for urinary tract infections in urogynaecological patients. Int. Urogynecol. J. Pelvic Floor Dysfunct. 19(5):671-676. 